\title{
Outpatient Parenteral Antimicrobial Therapy in Vulnerable Populations- People Who Inject Drugs and the Homeless
}

\author{
Alison Beieler, PA-C, MPAS'; Amalia Magaret, PhD 2,3,4; Yuan Zhou, MD; Anneliese Schleyer, MD ${ }^{1,4}$; \\ Anna Wald, MD, MPH ${ }^{2,4,6,7}$; Shireesha Dhanireddy, MD ${ }^{1,4 *}$
}

\begin{abstract}
${ }^{1}$ Harborview Medical Center, Seattle, Washington; ${ }^{2}$ Vaccine and Infectious Diseases Division, Fred Hutchinson Cancer Research Center, Seattle, Washington; ${ }^{3}$ Department of Biostatistics, University of Washington, Seattle, Washington; ${ }^{4}$ Department of Medicine, University of Washington, Seattle, Washington; ${ }^{5}$ The PolyClinic, Seattle, Washington; ${ }^{6}$ Department of Laboratory Medicine, University of Washington, Seattle, Washington; ${ }^{7}$ Department of Epidemiology, University of Washington, Seattle, Washington.
\end{abstract}

Outpatient parenteral antimicrobial therapy (OPAT) programs can provide high-value care but may be challenging in people who inject drugs (PWID) and homeless individuals. We conducted a single-center, retrospective, cohort study of adults who received OPAT at an urban, public health hospital from January 1, 2015 to April 30, 2016, grouped by PWID and housing status. Outcomes included clinical cure, length of stay, secondary bacteremia, line-tampering, and readmission. A total of 596 patients (homeless PWID (9\%), housed PWID (8\%), homeless non-PWID (8\%), and housed
non-PWID (75\%), received OPAT. Assuming that patients lost to follow-up failed therapy, homeless PWID were least likely to achieve cure compared with housed non-PWID, (odds ratio $[O R]=0.33,95 \% \mathrm{Cl} 0.18-0.59 ; P<.001$ ). Housed PWID were also less likely to achieve cure (OR $=0.37,95 \%$ $\mathrm{Cl} 0.20-0.67 ; P=.001)$. Cure rates did not differ in patients not lost to follow-up. OPAT can be effective in PWID and the homeless, but loss to follow-up is a significant barrier. Journal of Hospital Medicine 2019;14:105-109. (C) 2019 Society of Hospital Medicine

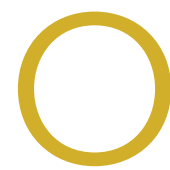

utpatient parenteral antimicrobial therapy (OPAT) programs allow patients to receive antibiotic therapy at home or in other settings. ${ }^{1-3}$ Bacterial infections among people who inject drugs (PWID) and the homeless are common, leading to complicated treatment strategies. Those with opioid dependence have frequent hospitalizations. ${ }^{4}$ Bacteremia and endocarditis frequently require intravenous (IV) antibiotics ${ }^{5-7}$ and may be difficult to treat. Creating outpatient treatment plans for PWID and the homeless is challenging, and there is a paucity of data on OPAT effectiveness in these groups as they are often excluded from OPAT services. ${ }^{1,2,8}$

We evaluated treatment outcomes in PWID and the homeless in our OPAT program.

\section{METHODS}

We conducted a retrospective cohort study of hospitalized adults discharged from Harborview Medical Center (HMC) with OPAT from January 1, 2015 to April 30, 2016. HMC is a county hospital in Seattle, Washington, affiliated with the University of Washington (UW). Infectious disease specialists

\footnotetext{
*Corresponding Author: Shireesha Dhanireddy, MD; E-mail: sdhanir@uw.edu; Telephone: 206-744-5103

Additional Supporting Information may be found in the online version of this article.
}

Received: May 16, 2018; Revised: October 11, 2018;

Accepted: December 3, 2018

(C) 2019 Society of Hospital Medicine DOI 10.12788/jhm.3138 supervise our OPAT program and provide follow-up care. We partner with a medical respite facility, a discharge option for homeless patients. ${ }^{9}$ Respite is staffed by HMC nurses, mental health specialists, and case managers.

Patients aged $\geq 18$ years were enrolled in OPAT if they were discharged with $>2$ weeks of IV therapy or required laboratory monitoring while on oral antibiotics. Patients with multiple hospitalizations were included for their initial OPAT encounter only. PWID discharged to respite were instructed not to use their vascular access to inject drugs, but drug abstinence was not required. A tamper-evident sticker was placed over lines that nurses evaluated daily. Patients violating line-tampering restrictions were discharged from respite, and OPAT providers developed alternative antibiotic plans.

The two primary exposures evaluated were patient-reported injection drug use and housing status, and our primary exposure measure was the four-category combination: (1) housed non-PWID, (2) housed PWID, (3) homeless non-PWID, and (4) homeless PWID. Current drug use was defined as use within three months of hospitalization. Homelessness was defined as lack of stable housing. Patients receiving chemotherapy, prolonged steroids, biologic agents, or those with organ transplant were considered immunocompromised.

The primary outcome was clinical cure, defined as completion of antibiotic therapy and resolution of infection, determined by OPAT providers. Patients who were placed on oral suppressive antibiotics or died before treatment completion were considered not cured. Unknown status, including care transfer and lost to follow-up, were noted separately. Lost to follow-up was assumed if patients did not return for care, their 
care was not formally transferred, and no other medical information was available.

Secondary outcomes included hospital length of stay (LOS), secondary bacteremia, line-tampering, and 30-day readmissions. Secondary bacteremia was defined as bacteremia with a different pathogen from the index illness, which occurred during the initial treatment course. Readmission included readmissions related to OPAT (ie, recurrent or worsening infection, treatment-related toxicities, line-tampering, secondary bacteremia, and line-associated complications).

Data collection was performed using REDCap, a data-capturing software program linked to the electronic medical record (EMR). ${ }^{10}$ Hospitalization dates and demographics were electronically populated from the EMR. Details regarding drug use, homelessness, comorbidities, diagnosis, discharge complications, clinical cure, and lost to follow-up were manually entered.

\section{Statistical Analysis}

Statistical calculations were performed using SAS (v. 9.4). Chisquare testing and analysis of variance were conducted to assess group differences in demographics, infection types, and clinical outcomes.

Primary and secondary outcomes were further evaluated by univariable logistic regression and presented as odds ratios, with the non-PWID housed group serving as the reference. Given the large number of PWID and homeless patients lost to follow-up, sensitivity analyses were conducted using the assumption that patients with unknown clinical outcomes did not achieve cure (ie, chronic infection or death). Multivariable regression was performed on the outcomes of cure and 30day readmission to OPAT using backward elimination to select a final model, initially including potential confounders of age, sex, and relevant comorbidities (DM and HIV). We assumed that those lost to follow-up were not cured (or readmitted). Other secondary outcomes were either rare events or those of uncertain relevance (eg, hospital LOS) to be evaluated in the multivariable analysis.

Our study did not meet the definition of research by the UW's institutional review board. It was a quality improvement project funded by a UW Medicine Patient Safety Innovations Program Grant.

\section{RESULTS}

Overall, 596 patients received OPAT over 16 months. OPAT patients were categorized into groups as follows: homeless PWID $(9 \%, n=53)$, housed PWID (8\%, $n=48)$, homeless non-PWID $(8 \%, n=45)$, and housed non-PWID (75\%, $n=450)$.

PWID were younger than non-PWID, and the majority of patients in all groups were men (Table 1). PWID were more likely to have hepatitis C. Non-PWID appeared more likely to have diabetes and be immunosuppressed.

Patients had a total of 960 types of infection (Table 1). Bacteremia was the most common infection among PWID. Osteomyelitis was the most frequent infection in non-PWID.

Discharge location varied widely $(P<.001$; Table 1). The majority of patients with housing (housed PWID 60.4\%, housed
non-PWID 59.1\%) were discharged to home, although $36.7 \%$ of housed non-PWID went to nursing facilities. Among homeless patients, $58.5 \%$ of PWID and $42.2 \%$ of non-PWID were discharged to respite; 10 patients were discharged to a shelter or street. Data specific to transition from IV to oral therapy were not recorded.

Cure rates among participants with known outcomes did not differ by group (Table $1 ; P=.85$ ). In a sensitivity analysis of clinical cure, assuming those with unknown outcomes were not cured, housing status and drug use were significantly associated with cure (Table $1 ; P<.001$, in the overall test), with rates lower among housed and homeless PWID groups $(50.0 \%$ and $47.2 \%$, respectively) compared with housed and homeless non-PWID groups (73.1\% and $82.2 \%$, respectively). In the multivariable analysis after backward elimination of noninfluential measures, only PWID and housing status were associated with cure; PWID, whether housed $(O R=0.37)$ or not $(O R=0.33)$, had lower odds of cure relative to housed non-PWID (Table 2).

Secondary outcomes, evaluated on all patients regardless of cure, differed by group (Table 1). Mean LOS appeared to be shortest for homeless PWID (15.5 days versus $\geq 18.0$ for other groups; $P<.001$ for overall test). Homeless PWID patients appeared more likely to have secondary bacteremia (13.2\% versus $<4.2 \%$ in other groups; $P<.001$ for overall test), line tampering ( $35.9 \%$ versus $<2.2 \%$ in other groups; $P<.001$ ), and 30 -day readmission related to OPAT $(26.4 \%$ versus $<16.7 \%$ in other groups; $P=.004$ ). Compared with housed non-PWID using logistic regression, homeless PWID had a higher risk of secondary bacteremia ( $\mathrm{OR}=12.9 ; 95 \% \mathrm{Cl}$ 3.8-37.8; $P<.001)$, line tampering (OR 88.4; 95\% Cl 24.5-318.3; $P<.001)$, and readmission for OPAT (OR 2.4; 95\% Cl 1.2-4.6; $P=.007$ ). After adjusting for age, sex, and comorbidities, readmission for OPAT remained elevated in homeless PWID $(O R=2.4$; 95\% Cl 1.24.6). No significant differences in secondary outcomes were found between housed non-PWID and also between housed PWID and homeless non-PWID.

Among homeless persons, discharge to respite care was not associated with improved outcomes, assuming those lost to follow-up did not achieve cure. Among non-PWID discharged to respite, the cure rate was $74 \%(14 / 19)$ compared with $88 \%$ $(23 / 26)$ discharged elsewhere $(P=.20)$. Among PWID, 48\% (15/31) discharged to respite were cured compared with $45 \%$ (10/22) discharged elsewhere $(P=.83)$.

\section{DISCUSSION}

Our study compares the outcomes of 596 OPAT patients, including PWID and the homeless. Among those retained in care, PWID achieved similar rates of cure compared with nonPWID groups. When assuming that all lost to follow-up had poor outcomes, the cure rates were markedly lower for PWID, with no difference noted by housing status.

Data on PWID and homeless enrolled in OPAT programs are limited. ${ }^{5,11,12}$ Few studies have reported the outcomes of infections in PWID and the homeless, as these populations often experience significant loss to follow-up due to transiency, lack of care continuity, and effective means of communication. 


\section{TABLE 1. OPAT Patient Characteristics $(N=596)$}

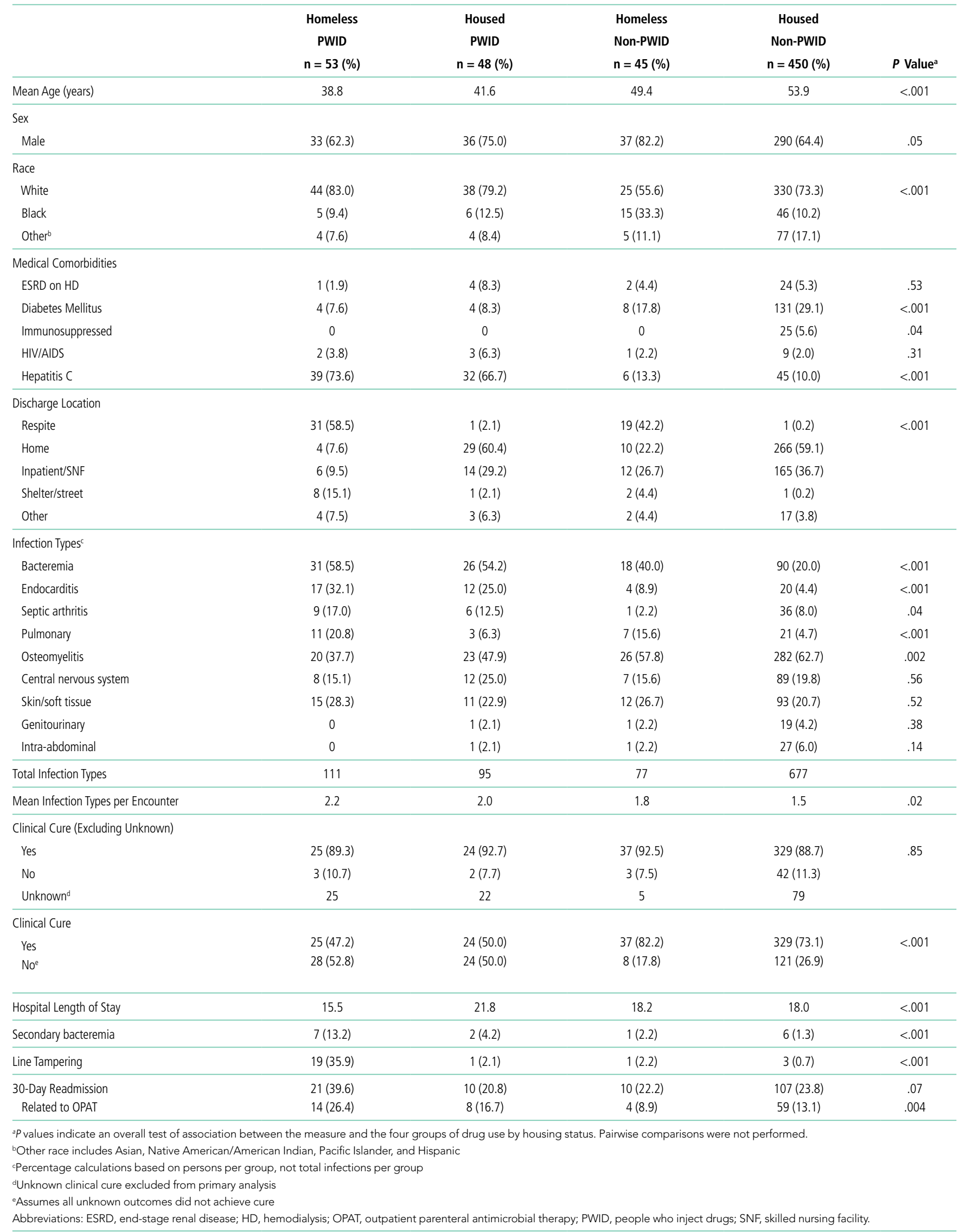


TABLE 2. Univariable and Multivariable Assessment of Factors Related to Cure. All 596 Persons are Included ${ }^{2}$

\begin{tabular}{|c|c|c|c|c|}
\hline Characteristic & Odds Ratio (95\% Cl) & $P$ Value & Adjusted Odds Ratio $(95 \% \mathrm{Cl})$ & $P$ Value \\
\hline \multicolumn{5}{|c|}{ Drug use and housing status } \\
\hline Homeless PWID & $0.33(0.18,0.59)$ & $<.001$ & $0.33(0.18,0.59)$ & $<.001$ \\
\hline Homeless non-PWID & $1.70(0.77,3.76)$ & .19 & $1.70(0.77,3.76)$ & .19 \\
\hline Housed non-PWID & Reference & & Reference & \\
\hline \multicolumn{5}{|l|}{ Sex } \\
\hline Women & Reference & & & \\
\hline Men & $0.81(0.56,1.18)$ & .28 & - & \\
\hline \multicolumn{5}{|l|}{ Comorbidities } \\
\hline Diabetes Mellitus & $1.23(0.81,1.85)$ & .34 & - & \\
\hline
\end{tabular}

"Those lost to follow-up prior to cure observation were included as "not cured."

Abbreviation: PWID, people who inject drugs

Cure was achieved in less than half of PWID, when lack of cure was assumed for unknown outcomes. This rate was substantially less than that for non-PWID groups. The assumption that those lost to follow-up did not achieve cure dramatically alters the inference; the truth may lie somewhere between the primary and sensitivity analyses. Homeless PWID remained at the highest risk for lost to follow-up, secondary bacteremia, line-tampering, and 30-day readmission related to OPAT.

PWID have traditionally been considered as a high-risk group for OPAT,1,2,8 but to completely restrict PWID from OPAT may not be appropriate. Ho et al. studied 29 PWID who were selectively enrolled to receive OPAT, and 28 completed IV therapy without any instances of line-tampering, death, or unknown clinical status. ${ }^{6}$ Recent literature suggests that some candidates can succeed with OPAT, despite drug use. ${ }^{13,14}$

Homelessness is also considered a barrier to OPAT. ${ }^{1,8}$ Medical respite is a harm-reduction model implemented for patients who require subacute care. ${ }^{9}$ In our study, among homeless patients, PWID status was the primary determinant of whether therapy was successful, rather than respite care.

Our study may have limited generalizability to other populations. We are a single-center facility in a large, urban city. PWID and housing status were self-reported but were verified before discharge. Most of our patients were men and white; thus, outcomes may differ for others. Due to the nature of the data, cost effectiveness could not be directly calculated. LOS and readmissions serve as proxy measures.

When patients remain engaged in care, PWID and the homeless achieved comparable clinical cure rates to those of housed non-PWID. Moving forward, OPAT can be more effective in PWID and the homeless with careful patient selection and close clinical support. Access to medication-assisted therapy, such as methadone or buprenorphine, ${ }^{15}$ may improve follow-up rates and linkage to outpatient care. Additional treatment strategies to improve retention in and adherence to care may promote successful outcomes in these vulnerable populations.

Disclosures: Presented at the Poster Abstract Session: Clinical Practice Issues at ID Week, October 26-30, 2016, New Orleans, LA. No conflicts of interested related to this work for all authors.

Funding: AW and AM received NIH NIAID grant K24 Al 071113-06 and UW

Medicine Patient Safety Innovations Program Grant.

\section{References}

1. Tice, AD, Rehm SJ, Dalovisio JR, et al. Practice guidelines for outpatient parenteral antimicrobial therapy. IDSA guidelines. Clin Infect Dis. 2004; 38(12):1651-1672. doi: 10.1086/420939.

2. Williams DN, Baker CA, Kind AC, Sannes MR. The history and evolution of outpatient parenteral antibiotic therapy (OPAT). Int J Antimicrob Agents. 2015;46(3):307-312. doi: 10.1016/j.ijantimicag.2015.07.001.

3. Gilchrist M, Seaton RA. Outpatient parenteral antimicrobial therapy and antimicrobial stewardship: challenges and checklists. J Antimicrob Chemother. 2015;70(4);965-970. doi: 10.1093/jac/dku517.

4. Ronan MV, Herzig SJ. Hospitalizations related to opioid abuse/dependence and associated serious infections from 2002-2012. Health Aff (Milwood). 2016;35(5):832-837. doi: 10.1377/hlthaff.2015.1424.

5. Beieler AM, Dellit TH, Chan JD, et al. Successful implementation of outpatient parenteral antibiotic therapy at a medical respite facility for homeless patients. J Hosp Med. 2016;11(8):531-535. doi: 10.1002/jhm.2597.

6. Ho J, Archuleta S, Sulaiman Z, Fisher D. Safe and successful treatment of intravenous drug users with a peripherally inserted central catheter in an outpatient parenteral antibiotic treatment service. J Antimicrob Chemother. 2010;65:2641-2644. doi: 10.1093/jac/dkq355.

7. Suleyman G, Kenney R, Zervos MJ, Weinmann A. Safety and efficacy of outpatient parenteral antibiotic therapy in an academic infectious disease clinic. J Clin Pharm Ther. 2017;42(1):39-43. doi: 10.1111/jcpt.12465.

8. Bhavan KP, Brown LS, Haley RW. Self-administered outpatient antimicrobial infusion by uninsured patients discharged from a safety-net hospital: a propensity-score-balanced retrospective cohort study. PLoS Med. 2015;12(12):e1001922. doi: 10.1371/journal.pmed.

9. Seattle-King County Medical Respite. https://www.kingcounty.gov/depts/ health/locations/homeless-health/healthcare-for-the-homeless/services/ medical-respite.aspx. Accessed October 2, 2018

10. Harris PA, Taylor R, Thielke R, et al. Research electronic data capture (REDCap) - a metadata-driven methodology and workflow process for providing translational research informatics support. J Biomed Inform. 2009;42:377- 
3781. doi: 10.1016/j.jbi.2008.08.010

11. Buerhle DJ, Shields RK, Shah N, Shoff C, Sheridan K. Risk factors associated with outpatient parenteral antibiotic therapy program failure among intravenous drug users. Open Forum Infect Dis.2017;4(3):ofx102. doi: 10.1093/ofid/ofx102.

12. Hernandez W, Price C, Knepper B, McLees M, Young H. Outpatient parenteral antimicrobial therapy administration in a homeless population. $J$ Infus Nurs. 2016;39(2):81-85. doi: 10.1097/NAN.0000000000000165.

13. Sukuki J, Johnson J, Montgomery M, Hayden M, Price C. Outpatient parenteral antimicrobial therapy among people who inject drugs: a review of the literature. Open Forum Infect Dis. 2018;5(9):ofy194. doi: 10.1093/ofid/ofy194. 14. D'Couto HT, Robbins GK, Ard KL, Wakeman SE, Alves J, Nelson SB. Outcomes according to discharge location for persons who inject drugs receiving outpatient parenteral antimicrobial therapy. Open Forum Infect Dis. 2018;5(5):ofy056. doi: 10.1093/ofid/ofy056.

15. Rosenthal ES, Karchmer AW, Theisen-Toupal J, Castillo RA, Rowley CF. Suboptimal addiction interventions for patients hospitalized with injection drug use-associated infective endocarditis. Am J Med. 2016;129(5):481-485. doi: 10.1016/j.amjmed.2015.09.024. 